\title{
Hip dysplasia among children with spastic cerebral palsy in rural Bangladesh
}

\author{
Tasneem Karim 1,2,3,4* ${ }^{*}$, Mahmudul Hassan Al Imam ${ }^{1,2}$, Prue Golland ${ }^{4}$, Aynul Islam Khan ${ }^{1,5}$, Iqbal Hossain ${ }^{6}$,
} Hayley Smithers-Sheedy ${ }^{4}$, Nadia Badawi ${ }^{3,4}$, Mohammad Muhit ${ }^{1,2}$ and Gulam Khandaker ${ }^{1,2,3,7}$

\begin{abstract}
Background: Hip dysplasia is common among children with cerebral palsy (CP), particularly in spastic CP. It can result in pain, reduced function and quality of life. However, the burden of hip dysplasia among children with CP in llow-and middle-income countries (LMICs) like Bangladesh is unknown. We aimed to define the burden of hip dysplasia among children with spastic CP in Bangladesh.

Methods: This study includes a subset of the Bangladesh CP Register (BCPR) study cohort who were registered between January and March 2015. The BCPR is a population-based surveillance of children with CP (aged $<18$ years) operating in a northern sub-district (Shahjadpur; child population 226,114) of Bangladesh. Communitybased key informant's method (KIM) survey conducted to identify children with CP in the surveillance area. A diagnosis of CP was made based on clinical history and examination by the study physicians and physiotherapist. Study participants had an antero-posterior (AP) X-ray of their pelvis. The degree of subluxation was assessed by calculating the migration percentage (MP).
\end{abstract}

Results: During the study period, 196 children with CP were registered, 144 had spastic CP. 40 children with spastic CP (80 hips) had pelvic X-Rays (mean age 9.4 years, range $4.0-18.0$ years) and $32.5 \%$ were female. Gross Motor Function Classification System (GMFCS) showed 37.5\% $(n=15)$ with GMFCS level I-II and 62.5\% $(n=25)$ with GMFCS level III-V. Twenty percent $(n=8)$ of the children had hip subluxation (MP: 33-80\%). Osteopenic changes were found in $42.5 \%(n=17)$ children.

Conclusions: To the best of our knowledge this is one of the first studies exploring hip dysplasia among children with spastic CP in Bangladesh. Our findings reflect that hip dysplasia is common among children with spastic CP. Introduction of hip surveillance programmes is imperative for prevention of secondary complications, reduced function and poor quality of life among these children.

Keywords: Hip dysplasia, Cerebral palsy, Children, Bangladesh, Surveillance

\section{Background}

Cerebral palsy (CP) is a heterogenous group of conditions that affects the developing brain, resulting in a permanent non-progressive dysfunction of the central nervous system manifested by disorders of motor function, movement, and posture [1]. It is the leading cause of childhood physical disability globally affecting two to three children per thousand live births [2, 3]. However, the burden of $\mathrm{CP}$ is even higher in low-and middle-

\footnotetext{
* Correspondence: tasneem.karim.tk@gmail.com

${ }^{1}$ CSF Global, Dhaka, Bangladesh

${ }^{2}$ Asian Institute of Disability and Development (AIDD), University of South

Asia, Dhaka, Bangladesh

Full list of author information is available at the end of the article
}

income countries (LMICs). Recent population-based studies from Bangladesh and Uganda reported population prevalence of $\mathrm{CP}$ as 3.4 and 2.9 per 1000 children respectively $[4,5]$.

The motor disorders of $\mathrm{CP}$ are often associated with musculoskeletal anomalies [6], of which hip displacement is the second most common abnormality preceded only by the abnormalities of the foot and ankle. It affects nearly one-third of the children with $\mathrm{CP}$ and the incidence varies from $1.0 \%$ in children with spastic hemiplegia, up to $75.0 \%$ in those with spastic quadriplegia $[7,8]$. Progressive hip displacement leading to dislocation can be catastrophic and result in extreme 
pain, reduced function and quality of life $[9,10]$. Other complications include severe contracture, pelvic obliquity and scoliosis, resulting in poor sitting, standing and walking abilities $[9,11,12]$. Most of the studies on hip dysplasia among children with $\mathrm{CP}$ are reported from high-income countries, and mostly from areas with an existing hip surveillance programme. However, there is limited data on hip dysplasia from LMICs such as Bangladesh where the burden of CP is substantially high [4].

Studies recommend that radiological screening of children with spastic quadriplegia [13] and GMFCS level IV and $V$ [14] should commence from less than 18 months of age [13]. It is also recommended that children with $\mathrm{CP}$ with a migration percentage (MP) of 33 to 40 should undergo radiological examination at 6 months interval prior to surgery [13]. Early detection and management of hip dysplasia can maintain flexible, properly located and pain-free hips with availability of symmetrical range of movement [15]. Introduction of formal surveillance programmes has demonstrated a significant reduction in the incidence of hip dislocation in populations of children with CP in high-income countries [14]. Hip dysplasia and dislocation are preventable through early identification and intervention [8]. However, the burden of hip dysplasia among children with CP in LMICs such as Bangladesh is unknown.

We aimed to describe the burden of hip dysplasia among a population-based cohort of children with $\mathrm{CP}$ in rural Bangladesh using the Bangladesh $\mathrm{CP}$ Register (BCPR) to inform clinicians and public health professionals [16].

\section{Methods}

\section{Study design, setting and participants}

This study is a part of the BCPR project. BCPR is one of the first population-based $\mathrm{CP}$ registers in a LMIC setting and has been ongoing since January 2015 in a northern part of Bangladesh i.e. Shahjadpur (child population 226,114) [17]. Children with CP registered with the BCPR were identified using a novel method, the Key Informant Method (KIM) which involves training of local volunteers to serve as key informants (KIs) and list children with $\mathrm{CP}$ from their community. The children then undergo detailed clinical assessment by a multidisciplinary team for confirmation of diagnosis and registration into the BCPR. The details of the BCPR study has been described previously $[4,16]$.

Between January and March 2015, a sub-group of the BCPR registrants were included in this study. Due to limited available resources, every third child from the BCPR cohort with spastic $\mathrm{CP}$ was selected for radiological assessment. In the event that any prospective study participant was unwilling the next child was approached for participation in the study.

\section{Clinical assessment}

Children with CP were identified from the community using the KIM, [18] and a detailed neurological examination was performed by a trained paediatrician and physiotherapist during the clinical assessment and $\mathrm{BCPR}$ registration process. Sociodemographic and clinical data were collected using BCPR record forms. Motor type and motor severity including Gross Motor Functional Classification System (GMFCS) levels were determined following standard guideline [19]. The predominant motor type of $\mathrm{CP}$ included spastic, dyskinetic or ataxic movement motor types based on clinical assessment by a paediatrician. Spastic topography was further classified as mono/hemiplegic, diplegic, triplegic, and quadriplegic.

\section{Radiological assessment}

Children with spastic $\mathrm{CP}$ had an anteroposterior (AP) pelvic X-ray. Hip displacement was measured from an AP radiograph of the hips using existing protocol [20]. The degree of subluxation (dysplasia) was assessed by calculating the MP i.e. the percentage of the bony width of the femoral capital epiphysis which falls lateral to a line drawn vertically from the bony lateral margin of the acetabulum, the Perkins line. This technique has been found useful to determine hip dysplasia among children [21]. Studies of validity have shown that a change greater than $8.3 \%$ in MP represents a real change in displacement of the femoral head with $95 \%$ confidence [20]. The MP found among the subjects were classified as normal hip (MP: <32\%), and subluxated hip (MP: $33-80 \%$ ) [22]. Radiological diagnosis of osteopenia was made by the radiologist after qualitative assessment of the $\mathrm{X}$ rays. Qualitative features of osteopenic bone included increased radiolucency, vertical striation, end plate thinning and accentuation of cortical margins of any vertebra including changes in shape.

\section{Ethical consideration}

The study was approved in Australia by the Cerebral Palsy Alliance NHMRC Human Research Ethics Committee (Ref no. 2015-03-02), and in Bangladesh by the Asian Institute of Disability and Development (AIDD) Human Research Ethics Committee (southasia-irb-2014-1-01) and Bangladesh Medical Research Council (BMRC) HREC (BMRC/NREC/ 2013-2016/1267). Informed written consent was obtained from the primary caregiver of the children prior to recruitment into the study.

\section{Statistical analysis}

The data were processed using SPSS (Statistical Package for Social Science, version 22.0 for windows; SPSS Inc., Chicago, IL, USA). The measures of central tendency (mean) and dispersion (SD) were used. Descriptive analyses were done to present the proportion of dysplastic hips according to the background factors (age, sex, 
parental education), and degree of motor impairment of the children with $\mathrm{CP}$.

\section{Results}

Out of the 196 BCPR registrants during the study period, 73.4\% $(n=144)$ had spastic CP of which 40 children underwent radiological assessment. The mean age was $9.4 \pm 4.0$ years (range $4.0-18.0$ years) and $32.5 \%(n=13)$ of the study cohort were female.

The majority of the mothers had completed primary education $(42.5 \%, n=17)$ whereas more than one third of the fathers had not have any education $(37.5 \%, n=15)$. The median age of diagnosis of $\mathrm{CP}$ was 3 years. The majority $(37.5 \%, n=15)$ of the children had diplegia. $37.5 \%$ $(n=15)$ were described as GMFCS level I-II and $62.5 \%$ $(n=25)$ as GMFCS level III-V. Large numbers of children with CP $(60 \%, n=24)$ had not ever received rehabilitation services. Twenty percent $(n=8)$ of the children had hip subluxation of which five children were between 5 to 9 years of age (Table 1).

Hip subluxation and age CP diagnosis, type of $\mathrm{CP}$, GMFCS and rehabilitation status

The majority $(75.0 \%, n=6)$ of the children who had subluxation of hips were diagnosed with $\mathrm{CP}$ between 24 to 47 months of age. Children with subluxated hips had spastic diplegia $(50.0 \%, n=4)$, quadriplegia $(37.5 \%, n=3)$ and hemiplegia/monoplegia $(12.5 \%, n=1)$. Children with GMFCS levels III -V had the highest proportion of subluxated hips; $37.5 \%(n=3)$. Among the children with hip subluxation $62.5 \%(n=5)$ never accessed any rehabilitation services (Table 2).

\section{Qualitative findings}

On qualitative assessment of the $\mathrm{x}$-rays by a radiologist we found that $42.5 \%(n=17)$ of the children had signs of osteopenic changes of which $94.1 \%(n=16)$ had mild osteopenia and 5.8\% $(n=1)$ had moderate osteopenic changes.

\section{Discussion}

The aim of the study was to investigate the burden of hip dysplasia among children with spastic CP from a population-based $\mathrm{CP}$ register in rural Bangladesh. We found that a fifth of our cohort had subluxation of hips which has yielded valuable insights into the high burden of hip dysplasia among children with spastic $\mathrm{CP}$ in Bangladesh.

Subluxation of hips was more commonly observed among children over 5 years of age and with more severe functional motor limitations (i.e. GMFCS levels III - V). This is consistent with other studies from high income countries which showed that the more severe outcomes of hip dysplasia are observed among older children and
Table 1 Socio-demographic characteristics, age of CP diagnosis, neurological subtype of Spastic CP, gross motor function classifications and rehabilitation status of children with CP

\begin{tabular}{|c|c|}
\hline Characteristics & $n(\%)$ \\
\hline \multicolumn{2}{|l|}{ Gender } \\
\hline Male & $27(67.5)$ \\
\hline Female & $13(32.5)$ \\
\hline \multicolumn{2}{|l|}{ Age (years) } \\
\hline$<5$ & $6(15.0)$ \\
\hline $5-9$ & $18(45.0)$ \\
\hline $10-14$ & $12(30.0)$ \\
\hline $15-18$ & $4(10.0)$ \\
\hline \multicolumn{2}{|l|}{ Maternal education } \\
\hline Illiterate & $11(27.5)$ \\
\hline Primary & $17(42.5)$ \\
\hline Secondary & $9(22.5)$ \\
\hline More than Secondary & $3(7.5)$ \\
\hline \multicolumn{2}{|l|}{ Paternal education } \\
\hline Illiterate & $15(37.5)$ \\
\hline Primary & $10(25.0)$ \\
\hline Secondary & $10(25.0)$ \\
\hline More than Secondary & $5(12.5)$ \\
\hline \multicolumn{2}{|c|}{ Age of diagnosis of CP (months) } \\
\hline $0-23$ & $1(2.5)$ \\
\hline $24-47$ & $21(52.5)$ \\
\hline $48-84$ & $11(27.5)$ \\
\hline $85 \&$ above & 7 (17.5) \\
\hline \multicolumn{2}{|l|}{ Spastic subtype } \\
\hline Hemi/monoplegia & $11(27.5)$ \\
\hline Diplegia & $15(37.5)$ \\
\hline Triplegia & $4(10.0)$ \\
\hline Quadriplegia & $10(25.0)$ \\
\hline \multicolumn{2}{|l|}{ GMFCS levels } \\
\hline Level I & $5(12.5)$ \\
\hline Level II & $10(25.0)$ \\
\hline Level III & $12(30.0)$ \\
\hline Level IV & $4(10.0)$ \\
\hline Level V & $9(22.5)$ \\
\hline \multicolumn{2}{|c|}{ Received rehabilitation services } \\
\hline Yes & $16(40.0)$ \\
\hline No & $24(60.0)$ \\
\hline
\end{tabular}

among those with higher GMFCS levels [7, 14, 23]. Furthermore, all the children with subluxation of hips in our cohort were diagnosed with CP after 24 months of age and nearly two thirds of them had not ever received any rehabilitation services. 
Table 2 Distribution of hip displacement according to socio-demographic factors, spastic CP sub-type, GMFCS and rehabilitation status

\begin{tabular}{|c|c|c|c|c|c|c|}
\hline & Right & & Left & & Total & \\
\hline Age & Normal & Subluxated & Normal & Subluxated & Normal & Subluxated \\
\hline $0-4$ years $(n=6)$ & $5(14.3)$ & $1(20.0)$ & $5(13.5)$ & $1(33.3)$ & $10(13.9)$ & $2(25.0)$ \\
\hline $5-9$ years $(n=18)$ & $14(40.0)$ & $4(80.0)$ & $17(45.9)$ & $1(33.3)$ & $31(43.1)$ & $5(62.5)$ \\
\hline $10-14$ years $(n=12)$ & $12(34.3)$ & $0(0.0)$ & $11(29.7)$ & $1(33.3)$ & $23(31.9)$ & $1(12.5)$ \\
\hline 15 and above $(n=4)$ & $4(11.4)$ & $0(0.0)$ & $4(10.8)$ & $0(0.0)$ & $8(11.1)$ & $0(0.0)$ \\
\hline \multicolumn{7}{|l|}{ Maternal Education } \\
\hline Illiterate $(n=11)$ & $11(31.4)$ & $0(0.0)$ & $11(29.7)$ & $0(0.0)$ & $22(30.6)$ & $0(0.0)$ \\
\hline Primary $(n=16)$ & $15(42.9)$ & $2(40.0)$ & $15(40.5)$ & $2(66.7)$ & $30(41.7)$ & $4(50.0)$ \\
\hline Secondary $(n=9)$ & $7(20.0)$ & $2(40.0)$ & $8(21.6)$ & $1(33.3)$ & $15(20.8)$ & $3(37.5)$ \\
\hline More than Secondary $(n=3)$ & $2(5.7)$ & $1(20.0)$ & $3(8.1)$ & $0(0.0)$ & $5(6.9)$ & $1(12.5)$ \\
\hline \multicolumn{7}{|l|}{ Paternal Education } \\
\hline Illiterate $(n=15)$ & $14(40.0)$ & $1(20.0)$ & $15(40.5)$ & $0(0.0)$ & $29(40.3)$ & $1(12.5)$ \\
\hline Primary $(n=10)$ & $9(25.7)$ & $1(20.0)$ & $9(24.3)$ & $1(33.3)$ & $18(25.0)$ & $2(25.0)$ \\
\hline Secondary $(n=10)$ & $7(20.0)$ & $3(33.3)$ & $9(24.3)$ & $1(33.3)$ & $16(16.7)$ & $4(50.0)$ \\
\hline More than Secondary $(n=5)$ & $5(14.3)$ & $0(0.0)$ & $4(10.8)$ & $1(33.3)$ & $9(12.5)$ & $1(12.5)$ \\
\hline \multicolumn{7}{|l|}{ Age of diagnosis of CP (months) } \\
\hline $0-23(n=1)$ & $1(2.9)$ & $0(0.0)$ & $1(2.7)$ & $0(0.0)$ & $2(2.8)$ & $0(0.0)$ \\
\hline $24-47(n=21)$ & $17(48.6)$ & $4(80.0)$ & $19(51.4)$ & $2(66.7)$ & $36(50.0)$ & $6(75.0)$ \\
\hline $48-84(n=10)$ & $10(28.6)$ & $1(20.0)$ & $10(27.0)$ & $1(33.3)$ & $20(27.8)$ & $2(25.0)$ \\
\hline $85 \&$ above $(n=7)$ & $7(20.0)$ & $0(0.0)$ & $7(18.9)$ & $0(0.0)$ & $14(19.4)$ & $0(0.0)$ \\
\hline \multicolumn{7}{|l|}{ Spastic subtype } \\
\hline Hemiplegia/ monoplegia $(n=11)$ & $11(31.4)$ & $0(0.0)$ & $10(27.0)$ & $1(33.3)$ & $21(29.2)$ & $1(12.5)$ \\
\hline Diplegia $(n=14)$ & $12(34.3)$ & $3(60.0)$ & $14(37.8)$ & $1(33.3)$ & $26(36.1)$ & $4(50.0)$ \\
\hline Triplegia $(n=4)$ & $4(11.4)$ & $0(0.0)$ & $4(10.8)$ & $0(0.0)$ & $8(11.1)$ & $0(0.0)$ \\
\hline Quadriplegia $(n=10)$ & $8(22.9)$ & $2(40.0)$ & $9(24.3)$ & $1(33.3)$ & $17(23.6)$ & $3(37.5)$ \\
\hline \multicolumn{7}{|l|}{ GMFCS } \\
\hline$|-|||(n=27)$ & $25(71.5)$ & $2(40.0)$ & $25(67.5)$ & $2(66.6)$ & $50(69.4)$ & $4(50.0)$ \\
\hline $\mathrm{IV}-\mathrm{V}(n=13)$ & $10(28.6)$ & $3(60.0)$ & $12(32.4)$ & $1(33.3)$ & $22(30.5)$ & $4(50.0)$ \\
\hline \multicolumn{7}{|l|}{ Received rehabilitation services } \\
\hline Yes $(n=24)$ & $23(65.7)$ & $1(20.0)$ & $22(59.5)$ & $2(66.7)$ & $45(62.5)$ & $3(37.5)$ \\
\hline No $(n=16)$ & $12(34.3)$ & $4(80.0)$ & $15(40.5)$ & $1(33.3)$ & $2(37.5)$ & $5(62.5)$ \\
\hline
\end{tabular}

Findings from our study have a bearing on the importance of hip surveillance among children with $\mathrm{CP}$ in LMIC settings. Hip dysplasia can be detected early by monitoring through regular hip X-rays to facilitate timely treatment and can thereby avert complications which are detrimental to the quality of life of children with $\mathrm{CP}$ and their families $[9,10]$. In contrast to high income countries, where a range of treatment options are available for the management of of children with $\mathrm{CP}$, including botulinum toxin injection, bracing, soft tissue release surgery for spasticity, reconstructive surgeries and salvage procedure [24], the prognosis of children with CP is remarkably different in poorer countries where there is limited access to such services. In addition to the lack of services these children and their families face additional challenges due to socioeconomic factors and rampant stigma around disabilities such as CP [25].

The position of femoral head within the acetabulum up to the age of 5 years is important to ensure stability of the hip and acetabular development [26, 27]. Therefore, early detection of progressively displaced hips and referral for orthopedic management is imperative to prevent further displacement and to keep the femoral head in position within the age of 5 years [28]. However, early diagnosis of hip dysplasia is challenging particularly through physical examination alone. Regular clinical 
inspection for the range of abduction of the hip, and repeated radiological examination of the hips is necessary to identify hip dysplasia in time $[8,29,30]$. The aim of hip surveillance programmes that are based on the Consensus Statement on Hip Surveillance for Children with Cerebral Palsy is to ensure that progressive hip displacement is detected early enough to enable timely referral for orthopedic assessment and management [31]. Decrease in incidence of hip dislocation has been previously documented with the introduction of hip surveillance programme [13]. Incorporation of hip surveillance into routine care has been recommended for all children with CP. This includes radiological assessment between 12 to 24 months of age with subsequent management varying with gross motor severity i.e. GMFCS levels [14]. Studies also suggested that non-ambulatory children and those who have an annual increment of the MP by more than $7 \%$ require more vigilant monitoring and consideration for orthopedic referral $[1,32]$.

A study conducted in Norway and Southern Sweden compared the burden of hip dislocation among children with $\mathrm{CP}$ in regions with and without hip surveillance programmes [20]. The sample size at the two sites $(n=119$ in Norway and $n=136$ in Southern Sweden) were comparable to our study cohort of 196 children with CP. The proportion of children at GMFCS levels III-V among both the study populations were substantially lower than our cohort; $34.0 \%$ in Norway and $38.0 \%$ in Southern Sweden population compared to $62.5 \%$ in our study cohort in Bangladesh [20]. It was observed that in Southern Sweden where hip surveillance services are provided the prevalence of hip dislocation was lower, fewer children required surgical intervention and the children underwent hip operations at an earlier age [20]. $20.0 \%$ of the children from our cohort had hip subluxation which is comparable to the findings from Norway (15.1\%). Whereas Southern Sweden had a significantly lower proportion $(0.7 \%, p<0.001)$ of children with hip subluxation in presence of a surveillance programme [20].

Early identification of hip dysplasia is limited by the absence of hip surveillance programmes, thereby resulting in a high burden [31,32]. A recent study conducted in India among 118 children with CP aged 2 to 12 years with GMFCS levels III-V showed that $12.7 \%$ had developmental dysplasia of spastic hip [21]. An even higher burden of hip dysplasia (54.7\%) was reported in another study conducted in Malaysia [33].

Findings from these studies collectively illustrate the apparent benefits of hip surveillance programmes in the reduction of the burden of hip dysplasia and the aversion of associated complications which drastically impair the wellbeing of children with CP [20, 21, 33]. There is a dire need for prospective hip surveillance programmes for children with CP in LMICs. Our study has direct clinical implication; the established programmes in high income countries and the method described in this study can be incorporated for the management of children with CP in LMICs. As radiological services are becoming increasingly available in semi-rural areas of LMICs such as Bangladesh, it is now possible to develop and implement hip surveillance programmes with a simplified protocol for these settings. Ongoing surveillance of children with $\mathrm{CP}$ such as the BCPR can serve as the basis for the implementation of recommended guidelines for hip dysplasia among children with $\mathrm{CP}$ in low resource settings. This will ensure early detection of hip dysplasia and subluxation which can thereby facilitate early intervention to avert and limit the adverse outcomes of hip dysplasia.

\section{Study limitations}

Despite our best efforts there are several limitations in this study which are inherent to observational studies. The cross-sectional design limited the establishment of causal relations between the independent variables and hip dysplasia. Radiography of hips were undertaken in a position as neutral as possible which was often difficult to achieve owing to spasm or contractures. We had to restrict or study only among children with spastic $\mathrm{CP}$ due to budget constraints. However, studies have shown that children with spastic $\mathrm{CP}$ are the most affected by hip dysplasia. Advanced statistical analyses were also limited due to the small number of children in subgroups (i.e. eight children with subluxation). Furthermore, caution is recommended in the interpretation of comparisons between studies due to methodological differences.

\section{Conclusion}

To the best of our knowledge this is one of the first studies exploring hip dysplasia among children with spastic $\mathrm{CP}$ in Bangladesh. Our findings from this selected sample suggest hip subluxation is common among children with spastic $\mathrm{CP}$ in rural Bangladesh. Further studies are needed to address the true extent of this complex complication of $\mathrm{CP}$ to improve the quality of life and ensure better prognosis among children with $\mathrm{CP}$ and their families. Introduction of hip surveillance programmes is imperative for the prevention of secondary complications, reduced function and poor quality of life among these children.

\section{Abbreviations \\ AP: Antero-posterior; BCPR: Bangladesh CP Register; CP: Cerebral Palsy; GMFCS: Gross Motor Function Classification System; KIM: Key Informant Method; Kls: Key Informants; LMICs: Low and Middle-Income Countries; MP: Migration Percentage}

\section{Acknowledgements}

We would like to acknowledge the CSF Global team in Bangladesh for their support in the implementation of the project and for supporting the children with CP and their families in access to services through a strong referral system. 


\section{Authors' contributions}

GK and MM conceptualized, designed and implemented the study. GK was responsible for collection of the data. AIK and $\mathrm{IH}$ reviewed the $X$-rays and provided specialist input. TK wrote the first draft of the manuscript with input from all co-authors. MHAI and GK contributed to data analysis and interpretation. MM, HSS, PG and NB provided specialist advice in the study. All authors read and approved the final manuscript and were involved in the decision to submit the manuscript.

\section{Funding}

This study has been conducted as part of the BCPR study. BCPR is funded by Cerebral Palsy Alliance Research Foundation (PG4314) and through internal funding from CSF Global, Bangladesh. The funding bodies played no role in the design of the study; collection, analysis, and interpretation of data and in the preparation of the manuscript. TK is supported by Cerebral Palsy Alliance Research Foundation Career Development Grant a (CDG 04617). HSS is supported through an NHMRC ECF 1144566 and the Australasian Cerebral Palsy Clinical Trials Network.

\section{Availability of data and materials}

The datasets generated and/or analysed during the current study are not publicly available due to confidentiality but are available from the corresponding author on reasonable request.

\section{Ethics approval and consent to participate}

The study was approved in Australia by the Cerebral Palsy Alliance Human Research Ethics Committee (Ref no. 2015-03-02), and in Bangladesh by the Asian Institute of Disability and Development (AIDD) Human Research Ethics Committee (southasia-irb-2014-I-01) and Bangladesh Medical Research Council (BMRC) HREC (BMRC/NREC/2013-2016/1267). Written informed written consent was obtained from the primary caregiver of the children prior to recruitment into the study.

\section{Consent for publication}

Not applicable

\section{Competing interests}

The authors declare that there is no competing interest.

\section{Author details}

${ }^{1}$ CSF Global, Dhaka, Bangladesh. ${ }^{2}$ Asian Institute of Disability and Development (AIDD), University of South Asia, Dhaka, Bangladesh. ${ }^{3}$ Discipline of Child and Adolescent Health, Sydney Medical School, The University of Sydney, Sydney, Australia. ${ }^{4}$ Cerebral Palsy Alliance Research Institute, The University of Sydney, Sydney, Australia. ${ }^{5}$ Singair Upazilla Health Complex, Singair, Manikganj, Bangladesh. ${ }^{6}$ Radiology and Imaging Department, Bangabandhu Sheikh Mujib Medical University (BSMMU), Dhaka, Bangladesh. ${ }^{7}$ Central Queensland Public Health Unit, Central Queensland Hospital and Health Service, Rockhampton, Queensland, Australia.

\section{Received: 8 April 2019 Accepted: 30 August 2019} Published online: 27 October 2019

\section{References}

1. Huser A, Mo M, Hosseinzadeh P. Hip surveillance in children with cerebral palsy. Orthop Clin North Am. 2018;49(2):181-90.

2. Ozmen M, Caliskan M, Apak S, Gokcay G. 8-year clinical experience in cerebral palsy. J Trop Pediatr. 1993;39:52-4.

3. Howard J, Soo B, Graham HK, Boyd RN, Reid S, Lanigan A, Wolfe R, Reddihough DS. Cerebral palsy in Victoria: motor types, topography and gross motor function. J Paediatr Child Health. 2005;41:479-83.

4. Khandaker G, Muhit M, Karim T, Smithers-Sheedy H, Novak I, Jones C, Badawi N. Epidemiology of cerebral palsy in Bangladesh: a populationbased surveillance study. Developmental Medicine \& Child Neurology. 2019; 61(5):601-9.

5. Kakooza-Mwesige A, Andrews C, Peterson S, Wabwire Mangen F, Eliasson AC, Forssberg H. Prevalence of cerebral palsy in Uganda: a populationbased study. Lancet Glob Health. 2017;5:e1275-82.
6. Rosenbaum P, Paneth N, Leviton A, Goldstein M, Bax M, Damiano D, Dan B, Jacobsson B. A report: the definition and classification of cerebral palsy April 2006. Dev Med Child Neurol Suppl. 2007 Feb 1;109(suppl 109):8-14.

7. Soo B, Howard J, Boyd R, Reid S, Lanigan A, Wolfe R, et al. Hip displacement in cerebral palsy. J Bone Joint Surg Am. 2006;88(1):121.

8. Miller F, Dabney KW, Rang M. Complications in cerebral palsy treatment. In: Epps Jr CH, Bowen JR, editors. Complications in pediatric orthopaedic surgery. Philadelphia: Lippincott Company; 1995. p. 477-544.

9. Bagg MR, Farber J, Miller F. Long-term follow-up of hip subluxation in cerebral palsy patients. J Pediatr Orthop. 1993;13:32-6.

10. Jung NH, Pereira B, Nehring I, Brix O, Bernius P, Schroeder SA, Kluger GJ, Koehler T, Beyerlein A, Weir S, von Kries R, Narayanan UG, Berweck S, Mall V. Does hip displacement influence health-related quality of life in children with cerebral palsy? Dev Neuro rehabil. 2014;17(6):420-5.

11. Muthusamy K, Chu H, Friesen R, Chou P, Eilert R, Chang F. Femoral head resection as a salvage procedure for the severely dysplastic hip in nonambulatory children with cerebral palsy. J Pediatr Orthop. 2008;28(8):884-9,

12. Moreau M, Drummond DS, Rogala E, Ashworth A, Porter T. Natural history of the dislocated hip in spastic cerebral palsy. Dev Med Child Neurol. 1979;21:749-53.

13. Hägglund $G$, Andersson S, Düppe $H$, Lauge-Pedersen $H$, Nordmark $E$, Westbom L. Prevention of dislocation of the hip in children with cerebral palsy. J Bone Joint Surg Br. 2005;87-B(1):95-101.

14. Wynter M, Gibson N, Willoughby KL, Love S, Kentish M, Thomason P, et al. Australian hip surveillance guidelines for children with cerebral palsy: 5-year review. Dev Med Child Neurol. 2015;57(9):808-20.

15. Dobson F, Boyd R, Parrott J, Nattrass G, Graham H. Hip surveillance in children with cerebral palsy. J Bone Joint Surg Br. 2002;84-B(5):720-6.

16. Khandaker G, Smithers-Sheedy H, Islam J, Alam M, Jung J, Novak I, et al. Bangladesh cerebral palsy register (BCPR): a pilot study to develop a national cerebral palsy (CP) register with surveillance of children for CP. BMC Neurol. 2015;15:173.

17. Bangladesh Bureau of Statistics. Population and housing census 2011: national volume 2- union statistics. Bangladesh: statistics and informatics division, Ministry of Planning. 2014. Available from: http://203.112.218.65: 8008/WebTestApplication/userfiles/Image/National\%20Reports/ Union\%20Statistics.pdf.

18. Mackey S, Murthy GV, Muhit MA, Islam JJ, Foster A. Validation of the key informant method to identify children with disabilities: methods and results from a pilot study in Bangladesh. J Trop Pediatr. 2012;58(4):269-74.

19. Rosenbaum PL, Palisano RJ, Bartlett DJ, Galuppi BE, Russell DJ. Development of the gross motor function classification system for cerebral palsy. Dev Med Child Neurol. 2008;50(4):249-53.

20. Elkamil Al, Andersen GL, Hägglund G, Lamvik T, Skranes J, Vik T. Prevalence of hip dislocation among children with cerebral palsy in regions with and without a surveillance programme: a cross sectional study in Sweden and Norway. BMC Musculoskelet Disord. 2011;12(1):284.

21. Vykuntaraju KN, Manohar V, Lakskman RR, Ramaswamy P. Developmental dysplasia of spastic hip in children with cerebral palsy in southern India. Indian Pediatr. 2016;53(3):259-60.

22. Pountney T, Green E. Hip dislocation in cerebral palsy. BMJ. 2006; 332(7544):772-5.

23. Terjesen $\mathrm{T}$. The natural history of hip development in cerebral palsy. Dev Med Child Neurol. 2012;54(10):951-7.

24. Shore B, Spence D, Graham $H$. The role for hip surveillance in children with cerebral palsy. Curr Rev Musculoskelet Med. 2012;5:126-34.

25. Benfer KA, Jordan R, Bandaranayake S, Finn C, Ware RS, Boyd RN. Motor severity in children with cerebral palsy studied in a high-resource and lowresource country. Pediatr. 2014;134(6):e1594-602.

26. Harris NH, Lloyd-Roberts GC, Gallien R. Acetabular development in congenital dislocation of the hip: with special reference to the indications for acetabuloplasty and pelvic or femoral realignment osteotomy. J Bone Joint Surg Br. 1975;57-B:46-52.

27. Kalen $\mathrm{V}$, Bleck EE. Prevention of spastic paralytic dislocation of the hip. Dev Med Child Neurol. 1985;27:17-24.

28. Scrutton D. The early management of hips in cerebral palsy. Dev Med Child Neurol. 1989;31:108-16.

29. Rang M. Cerebral palsy. In: Morrissy RT, editor. Lovell and Winter's pediatric orthopaedics. 3rd ed. Philadelphia: Lippincott Company; 1990. p. 465-506.

30. Scrutton D, Baird G. Surveillance measures of the hips of children with bilateral cerebral palsy. Arch Child. 1997;76:381-4. 
31. Wynter M, Gibson N, Kentish M, Love S, Thomason PK, Kerr Graham H. The consensus statement on hip surveillance for children with cerebral palsy: Australian standards of care. J Pediatr Rehabil Med. 2011;4(3):183-95.

32. Gordon G, Simkiss D. A systematic review of the evidence for hip surveillance in children with cerebral palsy. J Bone and Joint Surg Br. 2006; 88-B(11):1492-6.

33. Ching BH, Khoo TB. Prevalence and predictive factors of hip displacement in children with cerebral palsy at Paediatric institute, Kuala Lumpur hospital. Neurol Asia. 2017;1(3):22.

\section{Publisher's Note}

Springer Nature remains neutral with regard to jurisdictional claims in published maps and institutional affiliations.

Ready to submit your research? Choose BMC and benefit from:

- fast, convenient online submission

- thorough peer review by experienced researchers in your field

- rapid publication on acceptance

- support for research data, including large and complex data types

- gold Open Access which fosters wider collaboration and increased citations

- maximum visibility for your research: over $100 \mathrm{M}$ website views per year

At BMC, research is always in progress.

Learn more biomedcentral.com/submissions 